\title{
Endocrine Disrupting Chemicals: Sources, Effects And Treatments
}

\section{Muhammad Aqeel Ashraf*, Mesmire Wilson}

Department of Environmental Science and Engineering, China University of Geosciences, Lumo Road, Wuhan, China.

*Corresponding Author email: chemaqeel@gmail.com

\section{DOI: 10.2478/acmy-2019-0010}

\begin{abstract}
:
Hormonal changes in humans and animals can be attributed to endocrine disrupting chemicals (EDCs). Studies have found that excessive exposure to natural and artificial environmental chemicals and toxins can have adverse effects on the endocrine system and reproductive health. The endocrine system creates and releases hormones that regulate the development of organs and how they function. Any disruption to hormones affects the development and functioning of the reproductive system, the brain and the neurological system. Research and reports on the subject have been published by international experts and organizations including the World Health Organization (WHO), United Nations Environment Programme (UNEP), International Labour Organization (ILO) and Endocrine Society. To fully comprehend the effect of EDCs on humans and wildlife, it is essential to understand epigenetics and its transgenerational effects on hormone development. Here, we extensively explore and review the research on the sources of EDCs, their effects and why exposure to EDCs is of concern, and treatments for EDC exposure.
\end{abstract}

Keywords: EDCs; Hormones; Epigenetic; Receptors

\subsection{Introduction:}

For more than two decades, scientists have focused on the role of epigenetic changes in the production of altered phenotypes and diseases. Epigenetics refers to the changes in organisms caused by modifications or the mimicking of gene expression rather than alterations to the genetic code itself. Exposure to environmental factors, nutritional habits, xenobiotic chemicals, reproductive factors and low dose radiation alters epigenetic programming and concurrently enhances the risk of developing diseases. This paper focuses on the disruption caused by the resulting chemicals, also called endocrine disrupting chemicals (EDCs), in humans and/or wildlife.

EDCs are defined as chemicals that may interfere with the endocrine system and produce adverse developmental, reproductive, neurological and immune effects in both humans and wildlife [1]. In 1996, prior to this definition, the European Workshop in Weybridge, UK, defined an EDC as an "exogenous substance that causes adverse health effects in an intact organism, or its progeny, consequent to changes in endocrine function" [2]. The Endocrine Society defines an EDC as, "a compound, either natural or synthetic, which through environmental or inappropriate developmental exposures alters the hormonal and homeostatic systems that enable the organism to communicate with and respond to its environment". A thorough review of the literature on EDCs reveals that they can influence the organization of the developing brain, and EDC exposure may manifest in humans and wildlife as atypical social behaviors. Environmental exposure to EDCs increase susceptibility to various diseases and induces epigenetic alterations that can be transferred to subsequent generations. Exposure to environmental chemicals can cause adverse effects to developing organisms and can result in an increased risk of developing chronic diseases in adulthood. Natural and synthetic environmental EDCs can act as hormone mimics, disturbing developing organ systems and causing abnormalities that may not be apparent until much later in life [3].

Since 1995, the European Commission (EC) has actively taken actions against EDCs. In 1999, both the European parliament and the European council supported a community strategy on endocrine disrupters in three different phases. The short-term strategy focused on the establishment of a list of substances that require further evaluation for their role in endocrine disruption and on the use of existing legislation to control risk. Medium-term actions focused on the identification and assessment of endocrine disrupters and the research needed to better understand endocrine disruption. Long-term actions focused on legislative actions to protect human health and the surrounding environment [4].

The Inter-Organization Programme for the Sound Management of Chemicals (IOMC) was established in 1995 following recommendations made by the 1992 UN Conference on Environment and Development to strengthen co-operation and increase international co-ordination in the field of chemical safety. The participating organizations included the FAO, ILO, UNDP, UNEP, UNIDO, UNITAR, WHO, World Bank and OECD. The purpose of the IOMC is to promote coordination of the policies and activities pursued by the participating organizations, jointly or separately, to achieve the sound management of chemicals in relation to human health and the environment.

Following international recommendations in 1997 by the Intergovernmental Forum on Chemical Safety and the environment leaders of the participating organizations regarding the issue of endocrine disrupting chemicals (EDCs), the WHO, through the International Programme on Chemical Sa fety (IPCS), a joint program of WHO, UNEP and the International Labour Organization, developed a report in 2002 entitled Global Assessment of the State-of-the-Science of Endocrine Disruptors. Consequently, the Strategic Approach to International Chemicals Management (SAICM) was established by the International Conference on Chemicals Management (ICCM) in February 2006, to achieve the sound management of chemicals throughout their life cycle so that, by 2020 , chemicals will be used and produced in ways that minimize significant adverse effects on human health and the environment [1]

According to Bergman and his colleagues, SAICM recognizes that risk reduction measures need to be improved to prevent the adverse effects of chemicals on the health of children, pregnant women, fertile populations, the elderly, the poor, workers and other vulnerable groups and susceptible environments. They opined that one measure to safeguard the health of women and children is the minimization of chemical exposures before conception and through gestation, infancy, childhood and adolescence. "SAICM also specifies that groups of chemicals that might be prioritized for assessment and related studies, such as for the development and use of safe and effective alternatives, include chemicals that adversely affect, inter alia, the reproductive, endocrine, immune or nervous systems".

At its third session in September 2012, a resolution to include EDCs as an emerging issue under SAICM was adopted by the ICCM. EDCs are a challenge, as their effects depend on both the level and timing of exposure, which is especially critical when exposure occurs during development. EDCs may be released from the products that contain them, such as pesticides, flame retardants, plastic additives and cosmetics, which may result in residues or contaminants in food and other products [1]. According to the Endocrine Society, EDCs were originally thought to exert their actions primarily through nuclear hormone receptors, including estrogen receptors (ERs), androgen receptors (ARs), progesterone receptors, thyroid receptors (TRs), and retinoid receptors. Today, basic scientific research shows that the mechanisms are much broader than originally recognized.

"Thus, endocrine disruptors act via nuclear receptors, nonnuclear steroid hormone receptors (membrane ERs), non-steroid receptors (neurotransmitter receptors such as the serotonin receptor, dopamine receptor, and norepinephrine receptor), and orphan receptors, enzymatic pathways involved in steroid biosynthesis and/or metabolism, and numerous other mechanisms that converge upon endocrine and reproductive systems. Hence why, from a physiological perspective, an endocrine-disrupting substance is a compound, either natural or synthetic, which, through environmental or inappropriate developmental exposures, alters the hormonal and homeostatic systems that enable the organism to communicate with and respond to its environment" [5]. 


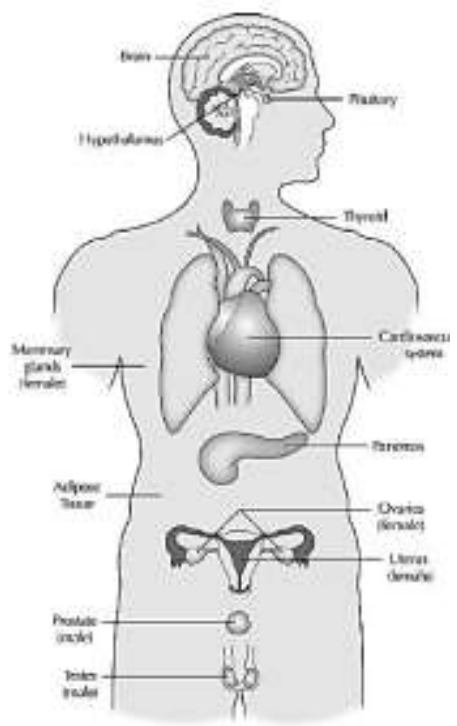

Figure 1: Model of the endocrine systems targeted by EDCs. All hormone-sensitive physiological systems are vulnerable to EDCs, including the brain and hypothalamic neuroendocrine systems, the pituitary gland, the thyroid gland, the cardiovascular system, the mammary glands, adipose tissue, the pancreas, the ovaries and uterus in females and the testes and prostate in males (The Endocrine Society).

Hypothalamus - The hypothalamus drives the endocrine system and links it to the nervous system.

Pituitary gland - The pituitary gland receives signals from the hypothalamus. The gland has two lobes, a posterior and an anterior lobe. The posterior lobe secretes hormones that are made by the hypothalamus. The anterior lobe produces its own hormones, several of which act on other endocrine glands.

Thyroid gland - The thyroid gland is critical to the healthy development and maturation of vertebrates and regulates metabolism.

Adrenal glands - The adrenal gland is made up of two glands: the cortex and medulla. These glands produce hormones in response to stress and regulate blood pressure, glucose metabolism, and the body's salt and water balance.

Pancreas - The pancreas is responsible for producing glucagon and insulin. Both hormones help regulate the concentration of glucose (sugar) in the blood.

Gonads - The male reproductive gonads, or testes, and the female reproductive gonads, or ovaries, produce steroids that affect growth and development and regulate reproductive cycles and behaviors. The major categories of gonad steroids are androgens, estrogens, and progestin, all of which are found in both males and females but at different levels.

\subsection{The transgenerational effects of epigenetics}

Parents pass on their genes to their children, and in so doing, pass on various traits associated with those genes. The combination of all genes in a species is referred to as the genome and genomic studies refer to studies designed to understand how various patterns of genes are controlled. Cells in the body can pass on heritable traits to their cellular progeny without altering their genomes [1]. During development, a single cell, the ovum, will divide, multiply and differentiate and, ultimately, become an adult. Development, from this perspective, is a process of fate restriction, permanently turning on or turning off different combinations of genes required for a cell to be functional in the liver, kidneys, brain, etc. This is an incomplete view of development. Instead, development is controlled not only by genetics but also by epigenetics and is subject to environmental changes.

Epigenetics is broadly defined as those heritable changes in the genome independent of changes to the genetic sequence, e.g., DNA methylation or histone modifications. It is these epigenetic processes that define and control tissue development by controlling gene expression. Thus, a major route by which hormones act during development is by changing the epigenome, i.e., the combination of genes that can or cannot be expressed. Though the mechanisms underlying these effects are a relatively new area of study, one manifestation of endocrine disruption is to alter a small subset of hormone-dependent epigenetic mechanisms and alter development [1].

Importantly exogenous chemicals have also been shown to produce heritable transgenerational effects as a result of their ability to alter epigenetic processes. This issue first arose with studies in which an anti-androgenic pesticide, vinclozolin, was given to developing mice at a time when the testes were in a critical period of development. Vinclozolin produced adverse effects on the developing testes and the effects were passed on to the following three generations of mice [6]. These effects were likely to be caused by epigenetic changes that were transmitted with high fidelity from one generation to the next via the germ cells. A number of exogenous chemicals have now been shown to influence epigenetic mechanisms and to produce effects in several generations of animals. Further studies should focus on this issue, but it is plausible that chemical exposures during pregnancy will affect the health of several subsequent generations of people and wildlife that are not themselves exposed [1].

The group of molecules identified as endocrine disruptors, according to Diamanti-Kandarakis, are highly heterogeneous and include synthetic chemicals used as industrial solvents/lubricants and their byproducts such as polychlorinated biphenyls (PCBs), polybrominated biphenyls (PBBs), and dioxins; plastics such as bisphenol A (BPA); plasticizers (e.g., phthalates); pesticides such as methoxychlor, chlorpyrifos, and dichlorodiphenyltrichloroethane (DDT); fungicides (e.g., vinclozolin), and pharmaceutical agents such as Diethylstilbestrol (DES).

Natural chemicals found in human and animal foods (phytoestrogens, including genistein and coumestrol) can also act as endocrine disruptors. These substances are generally thought to have a relatively low binding affinity to ERs but are widely consumed and are components of infant formula [7,8]. Urinary concentrations of the phytoestrogens genistein and daidzein were approximately 500 -fold higher in infants fed soy formula compared with those fed cow's milk formula [9]. Therefore, the potential for endocrine disruption by phytoestrogens needs to be considered. 
A challenge to the field of endocrine disruption is that these substances are diverse and may not appear to share any structural similarities other than usually being small molecular mass compounds (1000 Daltons). Thus, it is difficult to predict whether a compound may or may not exert endocrinedisrupting actions. Nevertheless, in very broad terms, EDCs such as dioxins, PCBs, PBBs, and pesticides often contain halogen group substitutions of chlorine and bromine [5]. "They often have a phenolic moiety that is thought to mimic natural steroid hormones and enable EDCs to interact with steroid hormone receptors as analogs or antagonists. Even heavy metals and metalloids may have estrogenic activity, suggesting that these compounds are EDCs as well as more generalized toxicants. Several classes of EDCs act as antiandrogens and as thyroid hormone receptor agonists or antagonists, and more recently, androgenic EDCs have been identified".

\subsection{What are hormones?}

To understand endocrine disruption, we must understand the basic features of the endocrine system; a series of ductless glands that secrete hormones directly into the blood to regulate various body functions. The traditional definition of a hormone is a molecule produced by an endocrine gland that travels through the blood to produce effects on distant cells and tissues [10]

"Traditional concepts of endocrine glands include the pituitary gland at the base of the brain, the thyroid gland in the neck, the adrenal glands in the abdomen next to the kidneys, the gonads and certain parts of the pancreas. In addition to these specialized endocrine glands, many other organs that are part of other body systems, such as the heart, body fat, muscle, liver, intestines, and kidneys, have secondary endocrine functions and also secrete hormones", as shown in Figure 1. Hormone effects are mediated by specific proteins called receptors. Without receptors, hormones cannot exert their effects. Steroid hormones tend to be carried through the blood by specific carrier proteins and are able to passively enter cells and interact with receptors inside the cells. In contrast, protein and amine hormones cannot passively enter cells, so specific mechanisms must be in place to allow these hormones to affect their target organs and cells; this usually involves interactions with cell membrane-associated receptors on the outside of the cell [10]. "Thyroid hormones are unique in that they act on receptors inside cells, but require specific transport proteins to gain access to the inside of a cell, unlike steroid hormones. We are learning that steroid hormones can also act through cell membrane receptors and this is likely to be important to fully under stand their effects and to understand the ways in which exogenous chemicals such as EDCs can interfere with their actions".

\subsection{Hormones control major physiological processes}

Hormones are important to both vertebrates and invertebrates. They are essential for controlling a large number of processes in the body, from early processes such as cell differentiation during embryonic development and organ formation, to the control of tissue and organ function in adulthood [10]. A well-known example is that of insulin, a small protein hormone produced by pancreatic beta cells.

These cells are stimulated to secrete insulin into the blood by the direct action of glucose. As blood glucose levels rise, during and after a meal, glucose enters the pancreatic beta cells through a specific protein transporter on the cell membrane and is converted inside the cells to the high-energy compound ATP. This process directly causes changes inside the beta cells, resulting in the secretion of insulin that was already produced and stored in anticipation of this event. Insulin travels through the blood to different tissues and cells, stimulating glucose uptake in the tissues via specific membrane receptors linked to transport systems. As a result, blood glucose levels fall, which shuts off insulin secretion from the beta cells. In this way, insulin is important, not only for maintaining glucose levels within a fairly narrow range in the blood but also for enabling glucose uptake and use by tissues as an energy source [10].

Insulin also reaches the brain, where it has important effects on appetite. Because insulin rises during and after a meal, it plays a role in hunger regulation. This is typical of hormones, they are primarily involved in important physiological processes but they also act on the brain to integrate various behaviors with specific physiological processes. Similarly, other hormones control major physiological functions and coordinate these functions between systems. Reproductive hormones, steroids (estrogens, androgens, progestins) and proteins (LH and FSH) control the complex physiological processes associated with reproduction. Thyroid hormones control metabolic processes and coordinate these with the many hormones involved in appetite and body weight regulation and metabolism. The adrenal hormones control the various physiological responses to stress.

In addition to their actions on these physiological processes, many hormones also control their own secretion by negative feedback. For example, thyroid hormone secretion is stimulated by the pituitary protein hormone, TSH (thyroid stimulating hormone), and thyroid hormones, in turn, suppress TSH. In this way, thyroid hormone levels are maintained within a relatively narrow range under normal circumstances. All hormone systems are governed, to some extent, by these processes so that blood hormone levels are at the appropriate concentration to be effective at controlling physiological processes. However, it is also important to note that there are times when tissues can control hormone action locally, such that the blood hormone level is not indicative of hormone action in the tissues [1].

"A wide variety of developmental problems and common adult diseases and disorders are caused by abnormal endocrine function. For example, diabetes is the result of a defect or defects in insulin action. Defects might be caused by the absence of insulin, insufficient or excessive amounts of insulin or by a defect or mutation in the insulin molecule or the receptors and proteins that mediate the actions of insulin action. Similar to most non-communicable diseases, diabetes is a result of a complex combination of genetic processes and the environment". Therefore, we need to measure and assess both types of factors to better understand complex diseases. The wealth of knowledge that has been gained by studying hormone systems and endocrine-related diseases has enhanced our ability to treat people with these diseases and forms the basis of our ability to identify environmental chemicals that can interfere with hormone action and to evaluate the consequences of exposure to these chemicals [1].

\subsection{Hormones act on receptors}

Hormones produce effects by acting on specialized proteins called receptors that attract and bind specific hormones. Hormone receptors provide specificity to hormone actions, both in terms of the time and the place of the hormone action. Hormone receptors are always limited in abundance and cause limited and specific effects downstream of hormone binding. Most hormones do not act in all cells because their receptors are not found in all cells. Most hormones also do not act at all times during the life cycle because their receptors are found only at specific times during development or in adulthood.

Importantly, hormones do not act the same way in all cells or in the same cell at different times during development and we are beginning to understand the physiology behind these differences. In some cases, multiple receptor types mediate the actions of a single hormone. For example, there are two receptor classes for thyroid hormones (TR $\alpha$ and TR $\beta$ ) and they have different temporal and spatial expression patterns. The spatial distribution of hormone receptors varies widely and accounts for the degree to which the hormone has global effects. For example, insulin receptors are found throughout the body and this accounts for the ability of insulin to affect all tissues. In contrast, TSH receptors are found predominantly in the thyroid gland. This limited distribution accounts for the much more restricted impact of TSH in the body. Likewise, estrogens exert their effects by acting on at least two major nuclear receptor types, estrogen receptor alpha $(E R \alpha)$ and estrogen receptor beta (ER $\beta)$, although they also act through specific membrane receptors on some cells. How many different kinds of receptors mediate estrogen actions is unknown [1].

In contrast, testosterone exerts its effects by acting on a single androgen receptor (AR). Despite the fact that some hormones act through multiple receptors and some hormones act through a single receptor, in all cases, the actions of a hormone are different in different cell types. There are important processes that contribute to the cell-specific nature of hormones and it is important to understand how this occurs so that the effects of environmental chemicals that interfere with these processes can be better predicted. In the case of nuclear receptors, receptors for steroid and thyroid hormones, the hormone-receptor complex binds to specific regions of DNA to regulate the process of gene transcription, resulting in the formation of new proteins. 
different receptor types in different cells, there are also mechanisms that allow the same receptor to have different effects in different cells. These mechanisms are not completely understood, but they include mechanisms that turn specific genes on or off independent of the hormone. Thus, a liver cell will produce different proteins than a brain cell, despite the fact that the same hormone, for example, estrogen, affects both cell types. Protein hormone receptors can also be located on the cell membrane.

Insulin, for example, binds to its membrane receptor and causes cells to take up and use glucose. After insulin binds to its receptor, there are very specific responses inside the cell that cause glucose uptake. It is important to recognize that insulin causes glucose uptake by different processes in different cells, even though there is only one insulin receptor. For example, insulin stimulates the production of glycogen in the liver (which sequesters glucose inside liver cells), but it activates a glucose transporter in other tissues, which directly stimulates glucose uptake. This occurs because the insulin receptor is linked to different kinds of cellular machinery in different cells. All protein and peptide hormones act in a similar fashion; there are specialized receptors on the outsides of the cells that transduce the effect of hormone binding to the inside of the cell.

These receptors are linked to different sets of proteins in different cells, which cause the cells to respond differently to the same hormone. Interestingly, there are also membrane receptors for some steroid hormones that also act via nuclear receptors. Estrogens and progestins both act through nuclear and membrane receptors. In these cases, the membrane receptor is coupled to fast-acting pathways that result in immediate effects, while the nuclear receptors take several hours to stimulate new protein production and exert effects [1]. Importantly, hormone receptors on the cell membrane can interact with hormone receptors in the nucleus by various mechanisms. Thus, different kinds of hormones can interact with each other through both extracellular and intracellular receptors to regulate development and various physiological processes.

\subsection{When do hormones act?}

Hormones are active throughout the stages of life (in utero, during infanthood, childhood, puberty, and adulthood). In fact, the timing of a hormone's action is an important determinant of its effect and potency. In adults, hormones have transient effects on target cells and tissues; the hormone has an effect when it is present but when the hormone is withdrawn the effect diminishes, similar to insulin levels rising when blood sugar is high and then declining when blood sugar is low. The transient effects of hormones in adults do not diminish their importance but contrast with their effects in the fetus and neonate where a hormone can have permanent effects in triggering early developmental events such as cell proliferation or differentiation [1].

During embryonic development, hormones cause some structures to develop (e.g., the male reproductive tract) and cause others to diminish (e.g., some sex-related brain regions). The changes produced by hormones at these critical times during development will last a lifetime. The actions of hormones during development are, in some ways, programming events. The term programming, in this case, refers to the ability of hormones to exert effects in the fetal period that influence or dictate the functions of the physiological systems in the adult. For example, the monthly cycle of changes in hormone levels in adult women that cause ovulation, and upon which female fertility depends, is programmed during fetal development by the actions of estrogens and androgens $[11,12]$.

Thus, small perturbations in estrogen levels during fetal development can change the reproductive axis in adulthood and diminish fertility [11]. Fetal programming events predispose the adult to a number of chronic diseases; thus, the prevention of endocrine diseases must begin with maternal and fetal health $[13,14]$. In some cases, studies have identified the developmental events that influence adult function, e.g., thyroid hormone and brain development.

During the fetal and neonatal periods in humans, thyroid hormone is essential for brain development, as the consequences of a lack of the hormone reveal in congenital hypothyroidism (CH) [15]. In fact, thyroid hormone is so important to development that all babies born in developed countries are universally screened for thyroid function to identify those children with a defective thyroid gland [15]. "This strategy has been successful in preventing severe mental retardation. Moreover, the fundamental knowledge we now have of the mechanisms by which thyroid hormone acts and the developmental events it controls can further guide clinical management of these and other thyroid disorders".

\subsection{Endocrine disruptors act on major physiological systems}

Endocrine disruptors interfere with the actions of hormones, and in doing so can produce adverse effects on the health of humans and wildlife. The physiological systems affected by EDCs include all the hormonal systems and EDCs can affect the development and function of the reproductive organs, adult-onset diabetes and cardiovascular disease. While there are many hormones and hormone systems, most studies of endocrine disruptors have focused predominantly on chemicals that interact with the estrogen, androgen and thyroid hormone systems.

A growing number of studies, however, indicate that environmental chemicals can interfere with other endocrine systems; no endocrine system can be excluded from being directly affected by environmental chemicals [16]. Fat development is a good example of a complex physiological system that is influenced by endocrine disruptors. There are a number of endocrine disruptors that affect weight gain, insulin sensitivity and glucose tolerance, indicating a potentially important role for endocrine disruptors in the development of obesity, type-2 diabetes and metabolic syndrome [16]. The elements of the endocrine system that control weight gain and metabolism/energy expenditure include the adipose tissue, pancreas, GI tract, liver, skeletal muscle, bone and brain, and endocrine disruptors can specifically and directly affect each of these tissues by interfering with their various hormone systems.

\subsection{Do endocrine disruptors act at low doses?}

Hormones act at low doses, in part by virtue of their strong affinity for their receptors. Some endocrine disruptors also have a very high affinity for nuclear receptors and as a result, can act at very low doses. Endocrine disruptors can act at low doses even if their affinity for the hormone receptors is considerably lower than that of the affinity of the native hormone for the receptor. This can happen, in part, because the impact of small changes in hormone action at the low end of the dose-response curve is much greater than at the high end of the dose-response curve. In addition, differences in receptor abundance have a large effect on the concentration of hormone (or endocrine disruptor) required to produce an effect [1]. "In addition, endocrine disruptors may have different potencies on different receptor isoforms (ER $\alpha$ or ER $\beta$ ). Therefore, the 'potency' of an endocrine disruptor will be highly dependent upon several important factors. This explains why some cells and tissues - or developmental time points - are much more sensitive to endocrine disruptors than others".

There are many examples of the low dose effects of endocrine disruptors [17]. The term low dose is defined in two ways. The first definition of a low dose is a dose much smaller than that which is traditionally accepted by toxicologists as the no adverse effect dose [18]. The second definition is that of a dose that is environmental relevant to humans [18]. One perspective is that chemicals exert toxicologically relevant adverse effects in a manner that is or approximates linearity, i.e., the dose makes the poison. In addition, the endpoints traditionally captured in toxicological studies are sufficient to determine all adverse outcomes [18]. In contrast, the low dose hypothesis posits that exogenous chemicals that interact with hormonal actions can do so in a manner that is specific such that traditional toxicological endpoints are not sufficient to preclude adverse outcome, and they do so with dose responses that are nonlinear and potentially non-monotonic [17].

\subsection{When do endocrine disruptors act?}

Endocrine disruptors act throughout the life cycle, just as hormones do, by interacting with the same pathways as hormones. When chemicals with endocrine disrupting activity are present during development, they affect cell programming and tissue development and their effects are typically permanent. When the same endocrine disruptor is present later in childhood or in adulthood, the effects are different and may be transient. The difference in the sensitivity and action of endocrine disruptors over the lifespan of an organism has several important implications. When human studies are designed to link chemical exposures to specific outcomes, it is important to measure chemical exposures at the developmental time-point that is appropriate for the specific outcome being measured [1]. 
Of course, the outcome may not be visible until adulthood in some cases. This may be more difficult for chemicals that do not persist in the body (many pesticides), than for chemicals that do (flame retardants, POPs). Another important implication is that not all endpoints of hormonal action will exhibit the same sensitivity to chemical exposure. The ability of endocrine disruptors to alter the normal hormonal control of development is perhaps the most significant consequence of EDC exposure because developmental effects occur at lower doses than are required for the same effects in adults [19].

Additionally, developmental EDC exposure has lifelong effects because EDCs affect cell differentiation and tissue development, resulting in tissues that have a different predisposition for disease in adulthood than that of non-exposed tissues. For example, a low dose of bisphenol A during fetal mouse development predisposes the prostate to the oncogenic actions of low doses of estrogen during adulthood, yet at birth, the prostate looks normal and the effect of BPA can only be detected by an -omics analysis [20].

\section{Occurrence of EDCs}

EDCs act at multiple sites via multiple mechanisms of action. Receptor-mediated mechanisms receive the most attention, but other mechanisms (e.g., hormone synthesis, transport, and metabolism) are equally important. For most reported associations between EDC exposure and the biological outcome, the mechanism(s) of action are poorly understood. This makes it difficult to distinguish between direct and indirect effects and primary versus secondary effects of EDC exposure. Considerable caution is necessary when extrapolating from in vitro data to in vivo effects, in predicting effects from limited in vivo data, and in extrapolating from experimental data to humans. A collective weight of evidence is essential in determining under what conditions the effects of EDC exposure. Despite an overall lack of knowledge of the mechanisms of action of EDCs, there are several examples where the mechanism of action is clearly related to direct perturbations to endocrine function and, ultimately, to adverse in vivo effects. These examples also illustrate important issues. Exposure to EDCs during the period when programming of the endocrine system is in progress may result in a permanent change of function or sensitivity to stimulatory/inhibitory signals.

i. Exposure in adulthood may be compensated for by normal homeostatic mechanisms and may therefore not result in any significant or detectable effects.

ii. Exposure to the same level of an endocrine signal during different life stages or during different seasons may produce different effects.

iii. Because of cross-talk between different components of the endocrine systems, effects may occur unpredictably in endocrine target tissues other than the system predicted to be affected. Considerable data are available on the early molecular events involved in the hormonal response, but there is little knowledge of the relationship between these molecular events and the potential for adverse health outcomes. Until such data become available, it will remain difficult and controversial to attribute adverse effects to endocrine-mediated pathways.

\section{Analysis of the Issues of Identifying and Measuring EDC Exposure}

Numerous chemicals in the environment (e.g., pesticides, industrial chemicals, and natural products) are hormonally active and they can be detected in people, wildlife and environmental samples. Some of these chemicals persist in the environment while others do not. Some are lipophilic, sequestered in adipose tissue and secreted in milk, and others are only present for short periods of time but at critical periods of development. Knowledge of the magnitude of EDC exposure in humans or wildlife remains limited. Most of the definitive studies on chemically mediated effects, including those on EDCs, have been conducted on highly exposed groups in various occupations or from accidental exposures. Because of analytical sensitivity and the latency in outcome after exposure, in only a few cases is appropriate low-level environmental exposure information available.

Hormonally-active environmental chemicals are extraordinarily diverse in their structure and potency. For example, some organ halogens, such as the PCBs, DDT, and PCDFs, are suspected endocrine-disrupting agents, but various members of these groups of chemicals exhibit profound differences in potency, biological and ecological persistence, and mechanisms of action. This kind of diversity creates obvious problems in human and ecological health assessments and increases the complexity and cost of chemical analyses in biological samples.

Synthetic chemicals are not the only exogenous agents that have caused health concerns because of their hormone-like activity. Of particular interest are the phytoestrogens (such as genistein and equol) and the fungal estrogens (such as zearalenone). The phytoestrogens and fungal estrogens are diverse in structure, undergo complex metabolic processes and are ubiquitous in the environment. They can be found in the blood and urine of virtually every person and animal on this planet, often at high concentrations. Phytoestrogens and fungal estrogens pose difficult analytical issues, yet, if phytoestrogen exposureresponse relationships remain uncertain, then the health assessments for many endocrine-disrupting agents, particularly environmental estrogens, will also remain uncertain.

This is because several of the phytoestrogens, mostly notably genistein and its analogs, possess binding affinities for the ER that are far greater than many of the EDCs of concern, such as the alkyl phenols, bisphenol A, and DDE. Phytoestrogens are far more potent and exert a far greater impact on humans than do synthetic chemicals. This does not mean that synthetic estrogens are of no concern. Instead, it emphasizes the need for EDC exposure assessments to consider both the magnitude of the exposure and the relative potencies of the array of EDCs that may have been encountered in the home, workplace, and general environment.

Information is needed to more accurately quantify the human, wildlife, and environmental burden of hormonally active environm ental chemicals so that quantitative comparisons can be made between body levels of natural and exogenous hormones based on potency, not just absolute amounts. This information is essential to properly evaluating exposure-response relationships in field and epidemiology studies and to using those relationships to produce credible risk assessments. Data on historical and geographical trends of EDC exposure are lacking. Knowledge of the fate and transport of new and existing chemicals is also limited, particularly among the different environmental compartments (water, sediment, and biota). Exposure assessment, particularly as it involves human health, must focus on vulnerable groups, in terms of both life stage and lifestyle. Exposure assessment for the critical development stages of gestation, lactation, adolescence, and senescence remains a high research priority.

The endocrine system, through a developmentally regulated pattern of expression, controls the pathways essential for cell proliferation, differentiation, and organ development, so it is not surprising that perturbations of the endocrine system during critical windows of sensitivity create the greatest potential for adverse health effects. The vulnerability of different groups in the population to EDC exposure will be affected by lifestyle factors (e.g., subsistence hunters and fishermen and avid sportsmen who consume fish and wildlife), genetic factors (e.g., metabolic differences that determine sensitivity), special dietary habits, and age (e.g., the types and rates of food consumption in children). Diet is likely the major EDC exposure route, however, an approach based on an integrated exposure assessment needs to be taken and all routes should be examined (e.g., dermal exposure, inhalation, and ingestion).

Examining the exposure of humans or wildlife to multiple chemicals that may function as EDCs (especially chemicals with a common mode of action and/or common target sites) is also critical. Exposure assessment encompasses both external measurements (levels in air, water, soil, food, etc.) and internal measurements (levels in blood, urine, and tissue samples). Both kinds of measurements provide critical information for wildlife, epidemiological, and experimental studies. Internal measurements are often confounded by the rapid metabolism of some EDCs [21]. This means that the quantification of metabolites or degradation products in biological samples is necessary for endocrine disruptor research. Some of the rapidly metabolized chemicals include the phthalate acid esters, alkyl phenols, diethylstilbestrol, some PCBs, phytoestrogens, and methoxychlor. Other complications to exposure assessment include time lags, seasonality, and multiple chemical exposures.

i. Time lags between EDC exposure and its effect: The transgenerational nature of some EDC effects may be the single most complicating factor. All of the potential latent effects that may occur from short-term exposures during critical development windows have not yet been identified. 
ii. Seasonality: Because of the sensitivity of the reproductive stages to EDCs, seasonality will be extremely important to wildlife. In addition, the association of EDCs with the aquatic environment is complicated by seasonal rainfall, storm runoff, and water releases.

iii. Multiple chemical exposures: This, is a factor for any toxic chemical, but it is especially important here because of the potential for effect modification (e.g., synergy, additivity, or antagonism).The most important need remains the continuation and improvement of environmental monitoring for the presence and quantity of contaminants.

Although environmental and tissue levels of certain EDCs (e.g., PCBs) have declined in some countries in response to regulations, they remain of concern in other countries, and uncertainty still exists regarding future trends. For most EDCs, data on trends are not available. Long-term data using harmonized collection and analysis methods are needed. Existing programs that furnish repeated measures of chemical contamination in the environment or in food provide our only indication of whether exposure is increasing or decreasing and to what magnitude.

\section{Ten Common Sources of EDCs}

\subsection{Personal Care Products}

Shampoos, conditioners, moisturizers, cosmetics, and other personal care products often contain endocrine disruptors, including (but certainly not limited to) phthalates. Phthalates are a group of gender-bending chemicals that cause the males of many species to become more female. Phthalates disrupt the endocrine systems of wildlife, including polar bears, deer, whales, and otters, causing testicular cancer, genital deformations, low sperm counts, and infertility. Another endocrine-disrupting chemical, triclosan, can even be found in certain brands of toothpaste. Switching to natural and/or homemade personal care products helps avoid EDC exposures. You can also try decreasing the number of personal care products used daily.

\subsection{Drinking Water}

Drinking water may be contaminated with atrazine, arsenic, and perchlorate, all of which may disrupt the endocrine system. Using a high-quality water filtration system, both at the tap and in the shower/bath, can help protect against EDC exposure. In many places, both surface water (rivers and lakes) and ground water are contaminated with EDCs from septic systems and treated waste water, storm water runoff, industrial waste, oil spills, agriculture, household products or fallout from the air. Most waste treatment facilities do not filter EDCs from sewage, and consequently, these chemicals are discharged into surface water.

\subsection{Canned Foods}

In an analysis of 252 canned food brands, 78 still use bisphenol-A (BPA), even though it is a known endocrine disruptor. BPA has been linked to a number of health concerns, particularly in pregnant women, fetuses and young children but also in adults. BPA coats approximately 75 percent of cans in North America, which means that if you eat canned foods, it is likely a major source of BPA exposure for you. Even BPA-free cans are not safe, as they are often coated with a similar chemical known as BPS. Buying products that come in glass bottles and jars rather than plastic or cans is ideal.

\subsection{Conventionally Grown Produce}

Pesticides, herbicides, and industrial runoff may coat conventionally grown fruits and vegetables in endocrine-disrupting chemicals. As much as possible, buy and eat organic produce and free-range, organic foods to reduce EDC exposure.

\subsection{CAFO Meat, Poultry, and Dairy Products}

Animals raised on concentrated animal feeding operations (CAFOs) also typically contain antibiotics, hormones, and other industrial chemicals that may disrupt your endocrine system. Look for animal products that are free-range, organic and raised on small, local farms that avoid the use of potential EDCs.

\subsection{High-Mercury Fish}

Fish contaminated with high levels of mercury and other heavy metals are problematic because such metals disrupt hormonal balance. Shark, swordfish, king mackerel, marlin, and tilefish are among the worst offenders but even tuna has been found to be contaminated with dangerously high levels of mercury and heavy metals. Farmed fish (the "CAFOS of the sea") also tend to be higher in contaminants and are best avoided. When eating seafood, smaller fish such as sardines, anchovies, and herring tend to be low in contaminants and high in omega-3 fats.

\subsection{Kitchen Products}

Plastic containers and non-stick cookware, common in many kitchens, are another source of EDCs. The plastic containers may contain BPA or other endocrine-disrupting chemicals that can leach into food, especially if the plastic is heated. Poly- and perfluoroalkyl substances (PFAS) used to create nonstick, stain-resistant, and water-repellant surfaces are also toxic and highly persistent both in the body and in the environment. When heated, non-stick cookware releases perfluorooctanoic acid (PFOA), which is linked to thyroid disease, infertility, and developmental and reproductive problems. Healthier options include ceramic and enameled cast iron cookware, both of which are durable, easy to clean (even the toughest cooked-on foods can be wiped away after soaking in warm water), and completely inert, which means they will not release EDCs.

\subsection{Cleaning Products}

Commercial solutions used to clean floors, toilets, ovens, and windows contain potential EDCs. For instance, nonylphenol ethoxylates (NPEs), a common ingredient in laundry detergents and all-purpose cleaners, is banned in Europe and known to be a potent endocrine disrupter, causing male fish to transform into females. It is surprisingly easy to create cleaning products using different combinations of vinegar, baking soda, essential oils, and coconut oil. Find simple tips for greener cleaning here.

\subsection{Office Products}

Ink cartridges, toner, and other solvents common in office environments are sources of endocrine-disrupting chemicals. Handle such products with care and minimize exposure as much as possible.

\subsection{Cash Register Receipts}

Thermal paper has a coating that turns black when heat is applied (the printer in a cash register applies heat to the paper, allowing it to print numbers and letters). Thermal paper contains BPA and handling thermal paper is enough to increase bodily levels of BPA. Holding the paper for just five seconds was enough to transfer BPA onto a person's skin, and the amount of BPA transferred increased approximately 10 times if fingers were wet or greasy. Finally, because receipts are often stored next to paper currency in people's wallets, paper currency may also be contaminated with BPA. In a study published in Environmental Science and Technology, researchers analyzed the paper currencies from 21 countries for the presence of BPA, and the chemical was detected in every sample.

\section{Why we should be concerned}

\subsection{Human and wildlife health depends on the ability to reproduce and develop normally}

Normal reproduction and development is impossible without a healthy endocrine system. The high incidence of and increasing trends of many endocrinerelated disorders in humans, observations of endocrine-related effects in wildlife populations, and the identification of chemicals with endocrine disrupting properties linked to disease outcomes in laboratory studies are fueling concerns over endocrine disruptors. 
13.2. Many endocrine-related diseases and disorders are on the rise

Large proportions (up to 40\%) of young men in some countries have low semen quality, which reduces their ability to father children. The incidence of genital malformations, such as non-descending testes (cryptorchidisms) and penile malformations (hypospadias), in baby boys has increased over time or leveled off at unfavorably high rates. The incidence of adverse pregnancy outcomes, such as preterm births and low birth weights, has increased in many countries. Neurobehavioral disorders associated with thyroid disruption affect a high proportion of children in some countries and have increased over past decades. Global rates of endocrine-related cancers (breast, endometrial, ovarian, prostate, testicular and thyroid) have been increasing over the past $40-50$ years.

There is a trend towards an earlier onset of breast development in young girls in all countries where this has been studied. Early breast development is a risk factor for breast cancer. The prevalence of obesity and type- 2 diabetes has dramatically increased worldwide over the last 40 years. The WHO estimates that 1.5 billion adults worldwide are overweight or obese and that the number of adults with type 2 diabetes increased from 153 million to 347 million between 1980 and 2008.

\subsection{Close to 800 chemicals are known or suspected to be capable of interfering with hormone receptors, hormone synthesis or hormone} conversion

Nevertheless, only a small fraction of these chemicals have been tested for their overt endocrine effects in intact organisms. The vast majority of chemicals in current commercial use have not been tested at all. This lack of data introduces significant uncertainties about the true extent of risks from potential EDCs.

\subsection{The rate at which disease incidence has increased in recent decades rules out genetic factors as the sole plausible explanation}

Environmental and other non-genetic factors, including nutrition, maternal age, viral diseases and chemical exposures, also affect disease risk but are difficult to identify. Despite these difficulties, some associations have become apparent: non-descended testes in young boys are linked with exposure to diethylstilbestrol (DES) and polybrominated diphenyl ethers (PBDEs) and with occupational pesticide exposure during pregnancy.

Recent evidence also shows that the painkiller paracetamol is linked to non-descended testes in young boys. However, there is little evidence that suggests that PCBs or dichlorodiphenyldichloroethylene (DDE) and DDT are associated with cryptorchidism. High exposure to polychlorinated dioxins and certain PCBs (in women who lack some detoxifying enzymes) are risk factors for breast cancer. Although exposure to natural and synthetic estrogens is associated with breast cancer, similar evidence linking estrogenic environmental chemicals with breast cancer is unavailable. The risk of prostate cancer is related to occupational exposure to pesticides (of an unidentified nature), to some PCBs and to arsenic. Cadmium exposure has been weakly associated with prostate cancer in some, but not all, epidemiological studies. Developmental neurotoxicity with negative impacts on brain development is linked to PCB exposure.

Attention deficit/hyperactivity disorder (ADHD) is overrepresented in populations with elevated exposure to organophosphate pesticides. Other chemicals have not been investigated. An excess risk of thyroid cancer was observed among pesticide applicators and their wives, although the nature of the pesticides involved was undefined.

\subsection{Significant knowledge gaps exist as to associations between EDC exposure and endocrine diseases}

There is little epidemiological evidence to link EDC exposure with adverse pregnancy outcomes, early breast development, obesity or diabetes. There is almost no information on the associations between EDC exposure and endometrial or ovarian cancer. High accidental exposures to PCBs during fetal development or to dioxins in childhood increase the risk of reduced semen quality in adulthood. With the exception of these studies, there are no data sets that include information about fetal EDC exposures and adult measures of semen quality. No studies have explored the potential link between fetal EDC exposure and the risk of testicular cancer occurring 20-40 years later.

\subsection{Numerous laboratory studies support the idea that chemical exposures contribute to endocrine disorders in humans and wildlife}

The most sensitive window of exposure to EDCs is during critical periods of development, such as during fetal development and puberty. Developmental exposures can cause changes that, while not evident as birth defects, can induce permanent changes that lead to the increased incidence of disease throughout life. These insights from endocrine disruptor research in animals have an impact on current toxicological testing and screening. Instead of solely studying the effects of EDC exposure in adulthood, the effects of EDC exposure during sensitive windows in fetal development, perinatal life, childhood and puberty require careful scrutiny.

13.7. Worldwide, there has been a failure to adequately address the underlying environmental causes of trends in endocrine diseases and disorders

Healthcare systems do not have mechanisms in place to address the contribution of environmental risk factors to endocrine disorders. The benefits that can be reaped by adopting primary preventive measures for dealing with these diseases and disorders have remained largely unrealized.

\subsection{Wildlife populations have been affected by endocrine disruption, with negative impacts on growth and reproduction}

These effects are widespread and have been due primarily to POPs. POPs bans have reduced exposure and led to the recovery of some wildlife populations. It is plausible that additional EDCs, which have been increasing in the environment and are of recent concern, are contributing to current population declines in wildlife species. Wildlife populations that are also challenged by other environmental stressors are particularly vulnerable to EDC exposures.

\subsection{Internationally agreed and validated test methods for the identification of endocrine disruptors capture only a limited range of the known} spectrum of endocrine disrupting effects

This increases the likelihood that harmful effects of EDCs in humans and wildlife are being overlooked. For many endocrine disrupting effects, agreed and validated test methods do not exist, although scientific tools and laboratory methods are available. For a large range of the effects of EDCs on human health, such as female reproductive disorders and hormonal cancers, there are no viable laboratory models. This seriously hampers progress in understanding the full scale of the risks of EDC exposure.

\section{Conclusions}

We are beginning to understand the importance of certain events during development and throughout the lifespan that interact with the genetic background to increase susceptibility to a variety of diseases. A large number of all non-communicable diseases have their origin during development. One of the important risk factors for disease is exposure to EDCs during development. Developmental EDC exposure results in an increased susceptibility to, and incidence of, a variety of diseases, as demonstrated in animal models and an increasing number of human studies. These include some of the major human diseases that are increasing in incidence and prevalence around the world. The incidence of these diseases and dysfunctions has increased at current levels of EDC exposure in normal populations. Human studies show that we are exposed to hundreds of environmental chemicals at any given time. It is now virtually impossible to identify a population around the globe that has been unexposed to EDCs.

There is an increasing burden of disease across the globe, in which EDCs likely play an important role and future generations will be affected. There have been clear benefits to human and wildlife health from the declining use of EDCs. Governmental actions to reduce exposure, while limited, have been effective in specific cases (e.g., bans and restrictions on lead, chlorpyrifos, tributyltin, PCBs and some other POPs) and have contributed to decreases in the frequency of disorders in humans and wildlife. The advances in our understanding of EDCs have been based mainly on information derived from studies in developed regions. There is still a major lack of data from large parts of the world, in particular from Africa, Asia and Central and South America. 
Better information on how and when EDCs act is needed to reduce developmental EDC exposure and prevent disease. A clear example of the success of primary prevention through exposure control is that of lead. To take advantage of our current knowledge to improve human and wildlife health by preventing environmentally induced diseases the following goals must be achieved.

\subsection{Strengthening knowledge of EDCs}

It is critical to move beyond the piecemeal, one chemical at a time, one disease at a time, one dose approach currently used by scientists studying animal models, humans or wildlife. Understanding the effects of the mixtures of chemicals to which humans and wildlife are exposed is increasingly important. The assessment of the action of EDCs on humans and wildlife by scientists needs to include the characteristics of the endocrine system that are being disrupted (e.g., low-dose effects and non-monotonic dose-response curves, tissue specificity and windows of exposure across the lifespan).

Interdisciplinary efforts that combine knowledge from wildlife, experimental animal model and human studies are needed to identify the chemicals that are responsible for the increased incidence of endocrine-related diseases and dysfunction. The known EDCs may not be representative of the full range of relevant molecular structures and properties because of the far too narrow focus on halogenated chemicals in many exposure assessments. Thus, a holistic approach is needed to identify other possible EDCs.

Endocrine disruption is no longer limited to estrogenic, androgenic and thyroid pathways. Chemicals also interfere with metabolism, fat storage, bone development and the immune system, which suggests that all endocrine systems can and will be affected by EDCs. Together, these new insights stress a critical need to acquire a better understanding of the endocrine systems to determine how EDCs affect normal endocrine function, how windows of exposure may affect the incidence of disease (particularly for childhood respiratory diseases) and how these effects may be passed on to future generations.

New approaches are needed to examine the effects of mixtures of endocrine disruptors on disease susceptibility and etiology, as an examination of endocrine disruptor in isolation is likely to underestimate the combined risk from simultaneous exposure to multiple endocrine disruptors. The asses sment of the effects of EDCs on human health needs to include an assessment of the effects of exposure to chemical mixtures on a single disease as well as the effects of exposure to a single chemical on multiple diseases. Since human studies, while important, cannot show cause and effect, it is critical to develop cause and effect data in animal studies to support the human studies.

\subsection{Reducing EDC exposure and susceptibility to disease}

It is imperative that we know the nature of EDCs to which humans and wildlife are exposed, together with information about their concentrations in the blood, placenta, amniotic fluid and other tissues, across life spans, gender, ethnicity (or species of wildlife) and regions. Many information gaps currently exist with regard to what types of EDCs are found in human and wildlife tissues, more so for developing countries and countries with economies in transition, and for chemicals that are less bioaccumulative in the body.

Long-term records that help us understand changes in exposures exist only for POPs and only for a few countries. In addition, there is a need to continue expanding the list of chemicals currently examined to include those contained in materials and goods as well as chemical by-products; it is impossible to assess exposure without knowing what chemicals to target. The comprehensive measurement of all exposure events during a lifetime is needed, as opposed to measurement at specific time points, and this requires longitudinal sampling, particularly during critical life stages, such as the fetal life stage.

\subsection{Identifying endocrine active chemicals}

Identifying potential EDCs from all the chemicals used and released worldwide is a major challenge, and we are currently only assessing the tip of the iceberg. It is possible to trace high production volume chemicals but not the numerous additives and processing chemicals used in low production volumes. Adding to the complexity and to the number of chemicals in our environment, are the unknown or unintended byproducts that are formed during chemical manufacturing and combustion processes and via environmental transformations. While the active ingredients in pharmaceuticals and pesticides have to be documented in the final product, this is not the case for chemicals used in materials and goods. Personal hygiene products and cosmetics require declarations of the ingredients because thousands of chemicals are used. Many sources of EDCs are unknown because of a lack of chemical constituent declarations in materials and goods. We need to identify sources of EDC exposure.

\subsection{Methods for evaluating evidence}

There is currently no consensual system for evaluating the strength of associations made between chemical exposures (including EDCs) and adverse health outcomes. Transparent methodologies are also missing. The need for developing better approaches for evaluating the strength of the associations, together with improved methods of risk assessment, is widely recognized. Methods for synthesizing the science into evidence-based decisions have been developed and validated in clinical arenas. However, because of differences between the environmental and clinical health sciences, the type of evidence and context of the decision used in these methods are not applicable to environmental contaminant exposures, including EDCs. To meet this challenge, it will be necessary to exploit new methodological approaches. It is essential to evaluate associations between EDC exposures and health outcomes by further developing methods for which proof of concept is currently under development.

\subsection{Improved testing for EDCs}

Validated screening and testing systems have been developed by a number of governments, and considerable time and effort are required to ensure that they function properly. These systems include both in vitro and in vivo endpoints and encompass studies in a variety of species, including fish, amphibians and mammals. Large batteries of high-throughput in vitro tests are being investigated for their ability to predict toxicity, the results of which may be used in hazard identification and risk assessment.

These new approaches are important as one considers the number of chemicals for which there is no information, and these high-throughput assays may provide important, albeit incomplete, information. An additional challenge is that, over the past decade, EDC research has revealed the complex interactions of some chemicals with endocrine systems, which may escape detection in current validated test systems. Finally, it will be important to develop weightof-evidence approaches that allow effective consideration of data from all levels of research, from in vitro mechanistic data to human epidemiological data.

\subsection{Creating enabling environments for scientific advances, innovation and disease prevention}

Exposure to EDCs and their effects on the health of humans and wildlife are a global problem that will require global solutions. More programs are needed that foster collaboration and data sharing among scientists and between governmental agencies and countries. To protect human health from the combined effects of EDC exposure, poor nutrition and poor living conditions, programs and collaborations between developed and developing countries and countries in economic transition are needed. New, adaptive approaches that break down institutional and traditional scientific barriers and stimulate interdisciplinary and multidisciplinary team science are needed.

\subsection{Ways to decrease the impact of environmental estrogens and xenoestrogens}

Whenever possible, leftovers should be stored in glass or ceramic containers instead of plastic ones. Avoid the use of the microwave to reheat food. Do not microwave foods stored in Tupperware or covered with Saran wrap, instead reheat in a pot on the stove. Choose biodegradable cleaning and laundry detergents or make your own non-toxic cleaning products at home. Do not use cleaning products that contain chlorine bleach. Avoid the use of fabric softeners, which place petrochemicals directly in contact with the skin. Buy hormone free/non-medicated meats or wild game (which is not treated with chemicals or antibiotics).

Do not eat farmed fish. Purchase organic, locally-grown produce that is free from pesticides, herbicides, chemical sprays or synthetic fertilizers or soak 
produce in a biodegradable vegetable wash for 10-15 minutes before consuming, which may remove small amounts of chemicals from the produce. Educate yourself about forms of natural pest control; remove all pesticides, herbicides, fungicides from the house, including insect repellants and weed killers for the lawn. Select unbleached recycled paper to use at the office or the house.

Purchase unbleached tampons or menstrual pads made with organic cotton that are free of surfactants, rayon and fragrance (the FDA detected dioxins and dozens of other substances in conventional tampons). Use essential oils and citronella lamps as insect repellants instead of toxic pesticides. Avoid condoms that contain the spermicide nonoxyl-9 that breaks down into nonylphenol and xenoestrogens. Avoid drinking tap water containing chlorine and other chemicals. Instead, drink reverse osmosis, bottled water. Consider investing in a filter for the shower to remove chlorine, which is readily absorbed through the skin, from the water. Do not let children chew on plastic toys. Choose non-bleached paper products including coffee filters, paper, napkins and toilet tissue.

\section{References}

[1] A. Bergman, Jerrold, J.J. Heindel, S. Jobling, K.A. Kidd, and R.T. Zoeller, "State of the Science of Endocrine Disrupting Chemicals- An Assessment of the State of the Science of Endocrine Disruptors Prepared by a Group of Experts for the United Nations Environment Programme (UNEP) and WHO", Geneva, Switzerland, 2012

[2] EC - European Commission. "Impact of endocrine disruptors on human health and wildlife", Weybridge, UK, 1996.

[3] R. Newbold, "Prenatal exposure to diethylstilbestrol (DES)", Fertility and sterility, Vol. 89, Pp. 55-56, 2008.

[4] M. Shahidehnia, "Epigenetic Effects of Endocrine Disrupting Chemicals", Journal of Environmental \& Analytical Toxicology, 2016.

[5] D.E.J. Bourguignon, L.C. Giudice, R. Hauser, G.S. Prins, A.M. Soto, R. Thomas Zoeller, and A.C. Gore, "Endocrine-Disrupting Chemicals: An Endocrine Society Scientific Statement", Endocrine Reviews, Vol. 30, No. 4, Pp. 293-342, 2009.

[6] M.K. Skinner, M. Manikkam, and C. Guerrero-Bosagna, "Epigenetic transgenerational actions of endocrine disruptors", Reproductive Toxicology, Vol. 31, No. 3, Pp. 337-343, 2011.

[7] G.G. Kuiper, J.G. Lemmen, B. Carlsson, J.C. Corton, S.H. Safe, P.T. van der Saag, B. van der Burg, and J.A. Gustafsson, "Interaction of estrogenic chemicals and phytoestrogens with estrogen receptor", Endocrinology, Vol. 139, Pp. 4252-4263, 1998.

[8] S.M. Dickerson, and A.C. Gore, "Estrogenic environmental endocrine-disrupting chemical effects on reproductive neuroendocrine function and dysfunction across the life cycle", Rev Endocr Metab Disord., Vol. 8, Pp. 143-159, 2007.

[9] Y. Cao, A.M. Calafat, D.R. Doerge, D.M. Umbach, J.C. Bernbaum, N.C. Twaddle, X. Ye, and W.J. Rogan, "Isoflavones in urine, saliva and blood of infantsdata from a pilot study on the estrogenic activity of soy formula", J. Expo. Sci. Environ Epidemiol., Vol. 19, Pp. 223-234, 2009.

[10] S. Melmed, and R.H. Williams, “Williams textbook of Endocrinology”, Philadelphia, PA, Elsevier/Saunders, 2011.

[11] M.M. Mahoney, and V. Padmanabhan, "Developmental programming: impact of fetal exposure to endocrine-disrupting chemicals on gonadotropinreleasing hormone and estrogen receptor mRNA in sheep hypothalamus", Toxicology and Applied Pharmacology, Vol. 247, No. 2, Pp. 98-104, 2010.

[12] H.N. Sarma, M. Manikkam, C. Herkimer, J. Dell'Orco, K.B. Welch, D.L. Foster, and V. Padmanabhan, "Fetal programming: exc ess prenatal testosterone reduces postnatal luteinizing hormone, but not folliclestimulating hormone responsiveness, to estradiol negative feedback in the female", Endocrinology, Vol. 146, No. 10, Pp. 4281-4291, 2005.

[13] A. Janesick, and B. Blumberg, "Endocrine disrupting chemicals and the developmental programming of adipogenesis and obesity", Birth Defects Research, Part C: Embryo Today--Reviews, Vol. 93, No. 1, Pp. 34-50, 2011.

[14] M.A. Hanson, and P.D. Gluckman, "Developmental origins of health and disease: moving from biological concepts to interventions and policy", International Journal of Gynaecology and Obstetrics, Vol. 115, No. 1, Pp. S3-5, 2011.

[15] R. Klein, "History of congenital hypothyroidism In :( Burrow GN, Dussault JH eds.)", Neonatal Thyroid Screening, pp. 51-59. New York, Raven Press, 1980.

[16] C. Casals-Casas, and B. Desvergne, “Endocrine disruptors: from endocrine to metabolic disruption”, Annual Review of Physiology, Vol. 73, Pp. 135-162, 2011.

[17] L.N. Vandenberg, T. Colborn, T.B. Hayes, J.J. Heindel, D.R. Jacobs, D.H. Lee, T. Shioda, A.M. Soto, F.S. vom Saal, W.V. Welshons, R.T. Zoeller, and J.P. Myers, "Hormones and endocrine-disrupting chemicals: Lowdose effects and nonmonotonic dose responses", Endocrine Reviews, Vol. 33, No. 3, Pp. 378-455, 2012.

[18] J.W. Owens, and J.G. Chaney, “Weighing the results of differing 'low dose' studies of the mouse prostate by Nagel, Cagen, and Ashby: Quantification of experimental power and statistical results", Regulatory Toxicology and Pharmacology, Vol. 43, No. 2, Pp. 194-202, 2005.

[19] P. Alonso-Magdalena, E. Vieira, S. Soriano, L. Menes, D. Burks, I. Quesada, and A. Nadal, "Bisphenol A Exposure during Pregnancy Disrupts Glucose Homeostasis in Mothers and Adult Male Offspring", Environmental Health Perspectives, Vol. 118, No. 9, Pp. 1243-1250, 2010.

[20] G.S. Prins, W.Y. Tang, J. Belmonte, and S.M. Ho, "Perinatal exposure to oestradiol and bisphenol A alters the prostate epigenome and increases susceptibility to carcinogenesis", Basic and Clinical Pharmacology and Toxicology, Vol. 102, No. 2, Pp. 134-138, 2008.

[21] R. Elsby, J.L. Maggs, J. Ashby, D. Paton, J.P. Sumpter, and B.K. Park, "Assessment of the effects of metabolism on the estrogenic activity of xenoestrogens: A two stage approach coupling human liver microsomes and a yeast estrogenicity assay", Pharmacology and Experimental Therapeutics, Vol. 296, No. 2, Pp. 329-337, 2001. 